Cita: Cid, L., Rodrigues, F., Santos Teixeira, D., Alves, J., Machado, S., Murillo-Rodríguez, E., \& Monteiro, D. (2020). Exploração de um modelo de segunda ordem da Versão Portuguesa da Basic Psychological Needs in Exercise Scale (BPNESp): validade do constructo e invariância. Cuadernos de Psicología del Deporte, 20(2), 95-111.

\title{
Exploração de um modelo de segunda ordem da Versão Portuguesa da Basic Psychological Needs in Exercise Scale (BPNESp): validade do constructo e invariância
}

\section{Exploración de un modelo de segundo orden de la versión portuguesa de la Escala de Necesidades Psicológicas Básicas en el Ejercicio (BPNESp): validez de constructo e invarianza}

\section{Exploration of a second order model of the Portuguese Version of Basic Psychological Needs in Exercise Scale (BPNESp): construct validity and invariance}

\begin{abstract}
Luis Cid 1,2, Filipe Rodrigues2,3, Diogo S. Teixeira4,5, José Alves1, Sérgio Machado6,7, Eric Murillo-
\end{abstract} Rodriguez6,8, Diogo Monteiro1,2

\begin{abstract}
1 Escola Superior de Desporto de Rio Maior (ESDRM-IPSantarém), Rio Maior, Portugal; 2. Centro de Investigação em Desporto, Saúde e Desenvolvimento Humano (CIDESD), Vila Real, Portugal; 3 Universidade da Beira-Interior (UBI), Covilhã, Portugal; 4 Universidade Lusófona de Humanidades e Tecnologias, Faculdade de

Educação Física e Desporto (ULHT/FEFD); ${ }_{5}$ Centro Interdisciplinar de Estudo da Performance Humana

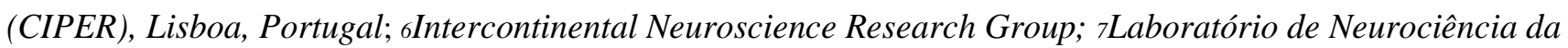

Atividade Física, Programa de Pós-graduação em Ciências da Atividade Física, Universidade Salgado de Oliveira, Niterói, Brasil; sLaboratory of Molecular and Integrative Neurosciences School of Medicine. Division of Health Sciences Anahuac Mayab University. Mérida, Yucatán. México
\end{abstract}

\begin{abstract}
RESUMO
O objetivo do presente estudo consistiu em analisar as propriedades psicométricas das versão portuguesa do Basic Psychological Needs Exercise Scale, testar um modelo de segunda ordem que represente as três necessidades psicológicas básicas, bem como analisar a invariância do modelo de medida de primeira e segunda ordem. Participaram neste estudo 550 sujeitos de ambos os géneros (264 femininos; 286 masculinos), com idades compreendidas entre os 14 e os 69 anos $(\mathrm{M}=30.0 ; \mathrm{SD}=10.2)$ praticantes de exercício físico em ginásios ou health clubs de diversas atividades. Os resultados revelaram que o instrumento possui validade de constructo (validade fatorial, convergente e discriminante), fiabilidade compósita e revelou ainda ser invariante em função do género, e atividades praticadas no contexto ginásio (musculação, cardiofitness e musculação), tanto para o modelo de $1^{\mathrm{a}}$ como de $2^{\mathrm{a}}$ ordem. Em suma, as evidências encontradas no presente estudo confirmam a validade e dão suporte à utilização sem reservas da versão Portuguesa da BPNESp para a avaliação das necessidades psicológicas básicas (individualmente ou através de um índice global) em investigações futuras no domínio do exercício
\end{abstract}




\title{
Cid, Rodrigues, Teixeira, Alves, Machado, Murillo-Rodríguez, Monteiro
}

Palavras chave: Análise Fatorial Confirmatória; teoria da autodeterminação; exercício; necessidades psicológicas básicas; invariância do modelo de segunda ordem

\begin{abstract}
The objective of this study was to reanalyze the psychometric properties of the Portuguese version of the Basic Psychological Needs Exercise Scale, assessing a second order model encompassing all three basic psychological needs. In addition, we examined measurement invariance for the first and second order model. This study included 550 subjects of both genders (264 female; 286 male), aged between 14 and 69 years $(\mathrm{M}=30.0 ; \mathrm{SD}=10.2)$, who practiced physical exercise at gymnasiums or health clubs of various activities. The instrument displayed construct validity (factor, convergent and discriminant validity), as well as acceptable composite reliability. Results showed measurement invariance between gender and gymnasium activities (i.e., bodybuilding, cardiofitness and weight training), in both first and second order models. In short, current evidence confirms the structure validity of the scale and supports the all-inclusive use of the BPNESp Portuguese version for assessing basic psychological needs, individually or as a global index, in future research in the field of exercise.
\end{abstract}

Key-words: Confirmatory Factor Analysis; Self-Determination Theory; exercise; basic psychological needs; secondorder model invariance

\section{RESUMEN}

El objetivo de este estudio fue analizar las propiedades psicométricas de la versión portuguesa de la Basic Psychological Needs Exercise Scale, probar un modelo de segundo orden que represente las tres necesidades psicológicas básicas, y analizar la invariabilidad del modelo de medición de primer y segundo orden. En este estudio participaron 550 sujetos de ambos sexos (264 mujeres; 286 hombres), de edades comprendidas entre los 14 y 69 años $(\mathrm{M}=30,0 ; \mathrm{SD}=10,2)$, que practicaban ejercicio físico en gimnasios o clubes de salud de diversas actividades. Los resultados mostraron que el instrumento tiene validez de constructo (validez de factor, convergente y discriminante), confiabilidad compuesta y también se encontró que es invariable de acuerdo al género, y actividades practicadas en el contexto del gimnasio (bodybuilding, cardiofitness y entrenamiento con pesas), tanto para los modelos de 1er y 2 do orden. En resumen, la evidencia encontrada en este estudio confirma la validez y apoya el uso sin reservas de la versión portuguesa del BPNESp para la evaluación de las necesidades psicológicas básicas (individualmente o a través de un índice global) en futuras investigaciones en el campo del ejercicio.

Palabras clave: Análisis factorial confirmatorio; teoría de la autodeterminación; ejercicio; necesidades psicológicas básicas; modelo de invariancia de segundo orden

\section{INTRODUÇÃO}

Quadro Conceptual

A Teoria da Autodeterminação (Self-Determination Theory) (SDT: Deci \& Ryan, 1985) é uma das mais populares e contemporâneas abordagens teóricas à motivação, que tem vindo a ser aplicada nas últimas três décadas, aos mais diversos contextos (e.g. educação, saúde, religião, política, trabalho) (ver: Deci \& Ryan, 1985; Ryan \& Deci, 2002), inclusive no contexto da atividade física (ver: Ryan \& Deci, 2007; Ryan, Williams, Patrick, \& Deci, 2009; Vallerand, Deci, \& Ryan, 1987; Vallerand \& Losier, 1999). De facto, nos últimos anos, temos vindo a assistir a uma proliferação dos estudos com base na SDT, no domínio da atividade física, nomeadamente: no desporto (e.g. Álvarez, Castillo, Duda, \& Balaguer, 2009; Gagné, Ryan, \& Bargmann, 2003; Luque et al., 2019; Monteiro et al. 2018a; Monteiro, Pelletier, Moutão, \& Cid, 2018b; Monteiro et al., 2019; Reinboth \& Duda, 2006), no exercício (e.g. Edmunds, Ntoumanis, \& Duda, 2006, 2008; Hagger \& Chatzisarantis, 2008; Markland \& Tobin, 2010; Rodrigues et al., 2018; Rodrigues et al., 2019; Rodrigues et al., 2020) e na educação física (e.g. Ntoumanis, 2001, 2005; Standage, Gillison, \& Treasure, 2007; Tristán et al., 2019).

A SDT é uma macro teoria que nos diz, segundo os seus autores (Deci \& Ryan, 1985, 2000; Ryan \& Deci, 


\section{Exploração da Versão Portuguesa da BPNES}

2007), que a motivação do sujeito não está diretamente influenciada pelos fatores do envolvimento social (e.g. clima motivacional das aulas, comportamento dos instrutores, clima motivacional dos treinadores), uma vez que a influência destes é mediada pela satisfação de três "nutrientes psicológicos inatos a todos os seres humanos e que são essenciais para o contínuo crescimento, integridade e bem-estar psicológico" (Deci \& Ryan, 2000, p. 229). Ou seja, as necessidades psicológicas básicas (BPN: Basic Psychological Needs) de autonomia (capacidade do sujeito regular as suas próprias ações) de competência (capacidade de eficácia do sujeito na interação com o envolvimento) e de relação (capacidade do sujeito de procurar e desenvolver ligações e relações interpessoais) (Ryan \& Deci, 2002) vão determinar a regulação do comportamento do sujeito, que assenta num continuum motivacional que oscila entre formas menos e mais autodeterminadas (i.e. controladas vs. autónomas). Segundo Ryan e Deci (2007), os sujeitos tendem a participar mais nas atividades quando regulam o seu comportamento por formas mais autodeterminadas e, por oposição, tendem a participar menos ou até mesmo abandonar, quando regulam o seu comportamento por formas menos autodeterminadas.

Em suma, podemos considerar que a SDT é uma abordagem à motivação alicerçada como meta teoria, que realça a importância dos recursos próprios que o ser humano tem na autorregulação do seu comportamento, que passa pela satisfação das necessidades psicológicas básicas de competência, autonomia e relação, pois são elas que estão na base do comportamento autodeterminado (regulação para formas mais intrinsecamente motivadas) (Ryan \& Deci, 2002). Qual é a importância deste facto? Aparentemente a resposta reside no facto de que "a motivação intrínseca pode estar entre os fatores mais importantes para a manutenção do exercício ao longo do tempo" (Ryan \& Deci, 2007, p. 5). De facto, a satisfação das necessidades inatas a todo o ser humano sustenta o comportamento intrinsecamente motivado, que por sua vez pode conduzir a resultados emocionais e comportamentais positivos (e.g. mais persistência, empenho, divertimento e prazer na atividade realiza) (Deci \& Ryan, 2000).

\section{Avaliação das Necessidades Psicológicas Básicas}

Para avaliar a importância das necessidades psicológicas básicas no contexto do exercício, bem como, a sua influência no comportamento dos sujeitos, é fundamental que exista um instrumento de medida específico para esse domínio, pois "a falta de instrumentos impede o progresso na compreensão do papel que as três necessidades têm na iniciação e manutenção do comportamento de exercício" (Vlachopoulos \& Michailidou, 2006, p. 182). Por essa razão, estes autores desenvolveram o Basic Psychological Needs in Exercise Scale (BPNES), um instrumento de medida para avaliar a satisfação das necessidades psicológicas básicas de autonomia, competência e relação no contexto do exercício), sendo constituído por 12 itens (4 itens cada fator).

A Basic Psychological Needs Exercise Scale (BPNES: Vlachopoulos \& Michailidou, 2006) foi inicialmente validado numa amostra de 504 sujeitos, de ambos os sexos, com idades compreendidas entre os 18 e os 65 anos, em praticantes de exercício em centros de fitness privados. Os resultados alcançados evidenciaram uma validade de constructo bastante satisfatória: $\left[\chi^{2}=122.3 ; \mathrm{df}=51 ; \mathrm{p}=<.001 ; \chi^{2} / \mathrm{df}=2.40\right.$; SRMR=.03; NNFI=.97; CFI=.97; RMSEA $=.05 ; 90 \%$ IC RMSEA=.04-.06], assim como, bons níveis de consistência interna ( $\alpha$ Autonomia $=.84$; $\alpha$ Competência $=.81 ; \alpha$ Relação=.92) e pesos fatoriais estandardizados entre 0.60 e 0.89 . Para além disso, o BPNES apresentou ainda uma elevada estabilidade temporal no teste-reteste sobre um período de quatro semanas.

Posteriormente, a BPNES foi igualmente validada numa amostra de 851 praticantes de exercício em programas comunitários (públicos) (Vlachopoulos, 2007), evidenciando também resultados bastante razoáveis no que se refere à validade de constructo: $\left[\chi^{2}=209.9 ; \mathrm{df}=51 ; \mathrm{p}=<.001 ; \chi^{2} / \mathrm{df}=4.12 ; \mathrm{NNFI}=.97\right.$; $\mathrm{CFI}=.98$; RMSEA=.06; 90\% IC RMSEA=.05-.07], à fiabilidade interna dos fatores ( $\alpha$ Autonomia $=.84$; $\alpha$ Competência $=.86 ; \quad \alpha$ Relação=.92) e aos pesos fatoriais dos itens (entre .59 e .90). Para além disso, o modelo de 3 fatores - 12 itens da BPNES também demonstrou ser invariante em função do género (Vlachopoulos, 2008) e em função do contexto da prática (centros privados vs. programas comunitários) (Vlachopoulos, 2007).

Em Portugal, a BPNES foi preliminarmente traduzida e validada para a população Portuguesa com recurso a uma análise fatorial exploratória (AFE) (Moutão, Cid, Leitão, \& Alves, 2008) e posteriormente confirmada com recurso a uma análise fatorial confirmatória 


\section{Cid, Rodrigues, Teixeira, Alves, Machado, Murillo-Rodríguez, Monteiro}

(AFC) (Moutão, Cid, Leitão, Alves, \& Vlachopoulos, 2009). Ambos os estudos foram realizados com base numa amostra de 410 sujeitos (276 femininos; 134 masculinos), com idades compreendidas entre os $13 \mathrm{e}$ os 80 anos $(\mathrm{M}=32.6 ; \mathrm{DP}=12.6)$, sendo estes praticantes de exercício físico em ginásios e health clubs.

Os resultados obtidos na AFE revelaram uma estrutura fatorial igual à versão original, explicando $57.3 \%$ da variância total dos resultados, com pesos fatoriais entre 0.58 e 0.82 , e uma consistência interna aceitável ( $\alpha$ Autonomia $=.64 ; \quad \alpha$ Competência $=.69$; $\alpha$ Relação=.81). No entanto, o item 9 , traduzido e construído para pertencer ao fator de autonomia apresentou um peso fatorial de 0.64 no fator da competência. Acrescido, este item estava a contribuir para a diminuição da consistência interna do suposto fator.

Os problemas encontrados com o item 9 refletiram-se igualmente na AFC, uma vez que o modelo só se ajustou aos dados de forma aceitável após a sua eliminação: $\left[\chi^{2}=142.2 ; \mathrm{df}=41 ; \mathrm{p}=<.001 ; \chi^{2} / \mathrm{df}=3.47\right.$; $\mathrm{CFI}=.92 ; \mathrm{RMSEA}=.08 ; 90 \%$ IC RMSEA $=.06-.09]$. De facto, o item 9 (“... eu sinto que a forma como faço exercício expressa verdadeiramente aquilo que sou." "... I feel that the way I exercise is a true expression of who I am."), apresentou uma correlação muito forte (cross-loading) com a necessidade psicológica básica de competência, o que indica que não estava a ser percecionado pelos sujeitos como um indicador exclusivo da satisfação de autonomia. Desta forma, os autores da versão Portuguesa (Moutão et al., 2009) aconselharam a revisão do seu conteúdo de modo a torná-lo mais compreensível e consistente, pelo que propuseram a seguinte composição: “... sinto que faço exercício de acordo com aquilo que verdadeiramente sou.".

Esta alteração foi posteriormente validada num estudo confirmatório do novo modelo (Moutão, Cid, Leitão, Alves, \& Vlachopoulos, 2012), com uma amostra de 522 praticantes de exercício (313 femininos; 209 masculinos), com idades compreendidas entre os $10 \mathrm{e}$ os 70 anos $(\mathrm{M}=32.8 ; \mathrm{DP}=12.1)$ e com diferentes níveis de envolvimento com a prática em termos temporais. Os resultados alcançados revelaram que a alteração efetuada ao item 9 produziu efeitos positivos que se refletiram num melhor ajustamento do modelo aos dados: $\quad\left[\mathrm{S}-\mathrm{B} \quad \chi^{2}=144.1 ; \quad \mathrm{df}=51 ; \quad \mathrm{p}=<.001 ; \quad \mathrm{S}-\mathrm{B}\right.$ $\chi^{2} / \mathrm{df}=2.82 ; \mathrm{NNFI}=.94 ; \mathrm{CFI}=.95 ; \mathrm{RMSEA}=.06 ; 90 \%$
IC RMSEA=.05-.07]. Para além disso, os valores do alfa de Cronbach evidenciaram igualmente uma melhoria na consistência interna dos três fatores, em especial nas necessidades psicológicas básicas de autonomia e competência ( $\alpha$ Autonomia $=.75$; $\alpha$ Competência $=.75$; $\alpha$ Relação=.83).

Tendo em consideração os resultados alcançados nos estudos previamente mencionados, o primeiro objetivo do presente trabalho consiste em analisar as propriedades psicométricas da versão Portuguesa da Basic Psychological Needs in Exercise Scale (BPNESp: Moutão, et al., 2009) com a alteração efetuada ao item 9 (Moutão et al., 2012). A revisão do instrumento será realizada através de uma análise fatorial confirmatória ao modelo de medida, numa nova amostra de sujeitos praticantes de exercício físico em ginásios e health clubs, de diversas atividades e com diferentes níveis de experiência. Pretende-se com o segundo objetivo validar um modelo hierárquico da BPNESp, de modo a obter um fator de $2^{\mathrm{a}}$ ordem que represente a satisfação da autonomia, da competência e da relação no seu conjunto, ou seja, um índice global de satisfação das necessidades psicológicas básicas no contexto do exercício. De acordo com a literatura teórica e empírica, acredita-se que o modelo hierárquico proposto possa ter um ajustamento adequado aos dados.

As razões de natureza teóricas estão relacionadas com os próprios fundamentos da SDT. Segundo Ryan e Deci (2000, 2002), existem determinadas condições que são essenciais ao ser humano para um funcionamento ótimo das suas disposições naturais necessárias para o crescimento, integração da personalidade, estruturas cognitivas do sujeito, bem como para o desenvolvimento social e bem-estar pessoal: a satisfação das necessidades psicológicas básicas (inatas e universais) de autonomia, competência e relação. Estas são "indicadores de uma necessidade psicológica básica global, de acordo com a hipótese de que a função motivacional ideal é alcançada com a satisfação de todas as três necessidades" (Ryan \& Deci, 2007, p. 7), ou seja, o modelo teórico preconiza que as três necessidades são essenciais no seu conjunto e que negligenciar qualquer uma delas terá consequências negativas. Em suma, "o bem-estar psicológico requer a satisfação de todas as três necessidades, satisfazer uma ou duas não é suficiente" (Deci \& Ryan, 2000, p. 229). 


\section{Exploração da Versão Portuguesa da BPNES}

As razões de natureza empírica estão relacionadas com o próprio instrumento de medida das necessidades psicológicas básicas no contexto do exercício. Se analisarmos os resultados das correlações entre os três 3 fatores subjacentes à BPNES, verificamos que os valores altos encontrados pressupõem a existência de um fator de $2^{\mathrm{a}}$ ordem. De acordo, com Kahn (2006), esta possibilidade deve ser fortemente equacionada quando existem correlações entre os fatores acima de 0.50 , o que se verifica na maioria dos estudos da validação da BPNES, nomeadamente: entre 0.48 e 0.76 (Vlachopoulos \& Michailidou, 2006); entre 0.63 e 0.76 (Vlachopoulos, 2007); e entre 0.53 e 0.84 (Vlachopoulos, 2008). O mesmo se passa em relação a versões da escala traduzidas para outras culturas, nomeadamente: entre 0.46 e 0.71 (versão Portuguesa: Moutão et al., 2009); e entre 0.45 e 0.72 (versão Espanhola: Sánchez \& Núñez, 2007).

Para além disso, a utilização de um índice global de satisfação das necessidades psicológicas básicas não é um procedimento inédito. De facto, esta estratégia já foi utilizada em diversos contextos para reduzir o número de parâmetros dos modelos a serem testados. Por exemplo, na educação (Gagné, 2003), no trabalho (Deci et al., 2001), e na educação física escolar (Ntoumanis, 2005) e no desporto (Monteiro et al., 2016). Inclusivamente, num dos estudos realizados com a BPNES (Vlachopoulos, 2007), o autor testou também um modelo hierárquico, com um fator de $2^{\mathrm{a}}$ ordem, obtendo resultados bastante satisfatórios: $\left[\chi^{2}=209.9 ; \mathrm{df}=51 ; \mathrm{p}=<.001 ; \chi^{2} / \mathrm{df}=4.12 ; \mathrm{NNFI}=.97 ;\right.$ CFI=.98; RMSEA=.06; 90\% IC RMSEA=.05-.07], o que na nossa opinião, vem reforçar a legitimidade do segundo objetivo do presente estudo.

Por último, o terceiro objetivo do presente estudo consiste em analisar a invariância do modelo de medida de $1{ }^{a}$ e de $2{ }^{a}$ ordem do BPNES em função do sexo e do tipo de modalidade praticada no contexto do ginásio e health clubs. Neste sentido, tendo por base as evidências teóricas descritas anteriormente (Ryan e Deci 2000, 2002), bem como as evidências empíricas anteriores (Vlachopoulos, 2007, 2008) e Vlachopoulos et al (2013) é expectável que os modelos sejam invariantes em função do género e das modalidades praticadas em ginásios e health clubs, neste caso concreto entre praticantes de musculação, cardiofitness e aulas de grupo.

\section{MATERIAL E MÉTODOS}

\section{Participantes}

Participaram neste estudo 550 sujeitos, de ambos os géneros (264 femininos; 286 masculinos), com idades compreendidas entre os 14 e os 69 anos $(\mathrm{M}=30.0$; $\mathrm{DP}=10.2$ ), praticantes de exercício físico em ginásios ou health clubs de diversas atividades (147 musculação; 175 aulas de grupo - indoor cycling, localizada, dança, step, yoga, combat, pump, jump; 231 cardiofitness - exercício que combina atividades aeróbias e musculação), com uma frequência de prática de 1 a 6 sessões por semana $(\mathrm{M}=3.1 ; \mathrm{DP}=1.1)$. Em termos de volume e experiência de treino, os participantes treinavam em média $4.9 \pm 2.4$ horas por semana (Min=1; Max=15) e tinham experiência de prática variava entre 1 e 240 meses ( $M=16.6$; $\mathrm{DP}=24.1)$.

\section{Instrumentos}

Basic Psychological Needs in Exercise Scale (BPNES: Vlachopoulos \& Michailidou, 2006). É um instrumento de medida constituído por 12 itens (4 itens por fator), aos quais se responde com base numa escala tipo Likert com 5 alternativas de resposta que variam entre o "Discordo Totalmente" (1) e o "Concordo Totalmente" (5). Posteriormente os itens são agrupados em três fatores (Autonomia, Competência e Relação), que representam as três necessidades psicológicas básicas subjacentes à SDT (SDT: Deci \& Ryan, 1985). No presente estudo iremos a utilizar a versão Portuguesa da escala (BPNESp: Moutão et al., 2009), com a alteração efetuada ao item 9 (Moutão et al., 2012).

\section{Procedimentos: Recolha dos Dados}

Em primeiro lugar, foram contactados os gestores e/ou administradores dos ginásios e health clubs sobre os propósitos do estudo, obtendo-se a permissão para recolher dados junto dos praticantes inscritos. Estes foram abordados de forma aleatória pelos investigadores e assistentes de investigação junto da área de receção antes da sessão de exercício, sempre ao final do dia, uma vez que é neste horário que existe uma maior afluência de praticantes nos ginásios. Após uma breve explicação sobre os objetivos do estudo, o instrumento de avaliação foi aplicado de forma individual, sempre em condições semelhantes. Para tal, teve-se o cuidado de criar as condições adequadas para que os indivíduos não se sentissem estranhos com 


\section{Cid, Rodrigues, Teixeira, Alves, Machado, Murillo-Rodríguez, Monteiro}

a situação e, ao mesmo tempo, pudessem estar concentrados durante o preenchimento do questionário. Todos os participantes que concordaram fazer parte do estudo fizeram-no de forma voluntária assinando o consentimento informado. Para além disso, foi garantida a confidencialidade dos dados recolhidos e assegurado que os mesmos não seriam, em momento algum, transmitidos a terceiros. O tempo de preenchimento do questionário demorou aproximadamente 10 minutos.

\section{Análise Estatística}

Inicialmente foram realizadas análises descritivas de medidas de tendência central (médias) e dispersão (desvio-padrão). Posteriormente foi realizada uma AFC de acordo com as sugestões de diversos autores (Byrne, 2010; Hair et al., 2014; Marsh, Hau, \& Wen, 2004;), sendo utilizado o método de estimação da Máxima Verosimilhança, através do teste do quiquadrado. Para demonstrar o ajustamento dos modelos de medida aos dados foram utilizados os tradicionais índices incrementais e absolutos: Comparative Fit Index (CFI), Tucker-Lewis-Index (TLI), Standardized Root Mean Square Residual (SRMR), Root Mean Square Error of Approximation (RMSEA) e o respetivo intervalo de confiança (90\% $\mathrm{IC})$, respetivamente. No presente estudo, para os índices referidos, foram adotados os valores de corte sugeridos por diversos autores (Byrne, 2010; Marsh et al., 2004; Hair et al., 2014): SRMR e RMSEA $\leq 0.08$, CFI e TLI $\geq 0.90$.

Por fim, foi analisada a validade convergente (i.e., se os itens convergem com os fatores), através do cálculo da Variância Extraída Média, considerando-se valores de corte $\geq 0.50$ (Fornell \& Larcker, 1981). De seguida, foi analisada a validade discriminante (i.e. avaliar se os fatores são suficientemente distintos uns dos outros), comparando o valor do quadrado da correlação entre os fatores com o valor de VEM de cada um dos fatores. Por último, foi calculada a fiabilidade compósita (FC) para avaliar a consistência interna dos fatores, adotando $\mathrm{FC} \geq 0.70$ como valor de corte, tal como sugerido por Hair et al. (2014). As análises foram realizadas com recurso aos programas de análise IBM SPSS STATISTICS 23.0 e AMOS 23.0 .

\section{Invariância Métrica}

A análise da invariância do modelo de medida entre género e modalidades praticadas foi realizada tendo em consideração os pressupostos recomendados por diversos autores (Byrne, 2010; Cheung \& Rensvold, 2002). Em primeiro lugar, o modelo de medida deve ajustar-se a cada grupo. Em segundo lugar, para executar uma análise de multi-grupos, devem ser examinados os seguintes tipos de invariância: invariância configural (i.e., modelo sem constrangimento); invariância métrica (igualdade dos pesos fatoriais); invariância escalar (igualdade dos covariâncias e pesos fatoriais) e; invariância residual (igualdade dos pesos fatoriais, covariâncias e erros de medida). Para a invariância do modelo de $2^{\mathrm{a}}$ ordem, foi retirado o critério de invariância escalar, uma vez que o modelo de $2^{\mathrm{a}}$ ordem não possui covariâncias e foi adicionado a invariância dos pesos das regressões entre o fator de $2^{\mathrm{a}}$ ordem e os fatores de $1^{\mathrm{a}}$ ordem. Os pressupostos de invariância foram verificados através das diferenças no CFI $(\Delta \mathrm{CFI} \leq .01)$ de acordo com as recomendações de vários autores (Byrne, 2010; Cheung \& Resnvold, 2002).

\section{RESULTADOS}

\section{Análise Preliminar}

Uma análise preliminar aos dados revelou que não existiram valores omissos nem outliers univariados e multivariados. Ou seja, não se verificaram problemas de distribuição univariada, uma vez que os valores de assimetria e achatamento, estavam compreendidos entre -2 e +2 e -7 e +7 , respetivamente (Byrne, 2010). No entanto, o coeficiente de curtose multivariado de mardia foi superior a $5.0 \mathrm{em}$ todos as amostras analisadas, indicativo de uma distribuição multivariada não normal (Byrne, 2010). Nesse sentido, seguindo as recomendações de Nevitt e Hancock (2001), foi aplicada a técnica de Bollen-Stine Bootstrap com 2000 amostras para as análises seguintes.

\section{Validade de Construto}

No que respeita à Tabela 1 , podemos verificar que os sujeitos valorizam mais os itens associados às necessidades psicológicas básicas de satisfação da autonomia e relação. Observa-se ainda que todos os pesos fatoriais variam entre 0.47 (competência) e 0.81 (relação), e que todos os fatores apresentaram consistência interna ajustada, obtendo-se valores de 


\section{Exploração da Versão Portuguesa da BPNES}

fiabilidade compósita entre .70 e .81. Relativamente à validade convergente, são evidenciados problemas nos fatores autonomia e competência (VEM <.50). No entanto, o fator relação apresentou validade convergente adequada (VEM $\geq .50$ ). Por fim, são evidenciados problemas de validade discriminante apenas entre o fator autonomia e competência (r2 $>$ VEM). Os restantes fatores não apresentaram problemas de validade discriminante ( $\mathrm{r} 2<\mathrm{VEM})$.

Na Tabela 2 e 3 é possível observar-se que tanto o modelo de medida de $1^{\mathrm{a}}$ ordem como de $2^{\mathrm{a}}$ ordem se ajustaram aos dados em todas as amostras em análise, conforme os valores de corte adotados na metodologia.

No que respeita aos resultados apresentados na Figura 1 referentes ao modelo de $2^{\mathrm{a}}$ ordem (i.e., 1 fator de $2^{\mathrm{a}}$ ordem $\rightarrow 3$ fatores de $1^{\text {a }}$ ordem e 12 itens), verifica-se que existem relações positiva significativas, $(\beta=.83$; $\beta=.74 ; \beta=.64)$, entre o fator de $2^{\mathrm{a}}$ ordem e as três necessidades psicológicas básicas. Para além destas relações o fator compósito apresentou um valor de consistência interna de 0.80 , considerando-se como ajustado.

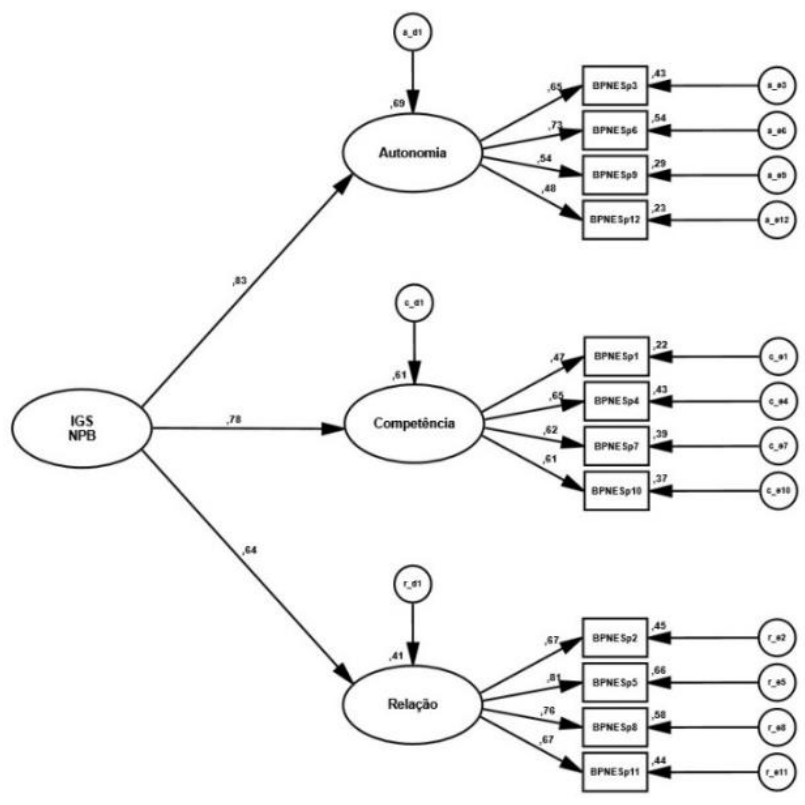

Figura 1. Parâmetros individuais estandardizados do modelo de $2^{\mathrm{a}}$ ordem da BPNESp ( 1 fator de $2^{\mathrm{a}}$ ordem; 3 fatores de $1^{\text {a }}$ ordem e 12 itens)

\section{Invariância Métrica}

Na Tabela 4 e 5 estão dispostos os modelos relativamente à análise multi-grupos em função do género e das diferentes modalidades do ginásio, respetivamente. Os resultados revelaram que ambos os modelos (i.e. $1^{\mathrm{a}}$ e $2^{\mathrm{a}}$ ordem) são invariantes $(\Delta \mathrm{CFI}<.01)$.

\section{DISCUSSÃO}

Os objetivos do presente estudo consistiram, primeiramente, em analisar as propriedades psicométricas da versão portuguesa da BPNE (Moutão et al., 2009), tomando em consideração a modificação feita ao item 9 (Moutão et al., 2012). De seguida, pretendeu-se validar um modelo hierárquico de $2^{\mathrm{a}}$ ordem de forma a obter um único fator global que representasse a satisfação das necessidades psicológicas básicas. Por último, pretendeu-se analisar a invariância em função do género e do tipo de atividade praticada no ginásio do modelo de $1^{\mathrm{a}}$ e $2^{\mathrm{a}}$ ordem, alargando desta forma as evidências científicas. Resultados do presente estudo contribuem para aquilo que Deci e Ryan (2008) designaram de desenvolvimento de conhecimento sobre a universalidade das variáveis subjacentes à Teoria da Autodeterminação, que neste caso concreto diz respeito à satisfação das necessidades psicológicas básicas. Os resultados revelaram que o instrumento possui validade de constructo (validade fatorial, convergente e discriminante), fiabilidade compósita e revelou ainda ser invariante em função do género, e atividades praticadas no contexto ginásio (aulas de grupo, cardiofitness e musculação), tanto para o modelo de $1^{\mathrm{a}}$ como de $2^{\mathrm{a}}$ ordem. 


\section{Cid, Rodrigues, Teixeira, Alves, Machado, Murillo-Rodríguez, Monteiro}

\section{Tabela 1.}

Parâmetros Individuais Estandardizados dos Itens da BPNESp, fiabilidade compósita, validade convergente e discriminante

\begin{tabular}{lcccccccc}
\hline Item (Factor) & Mín-Máx & M \pm DP & FL & FC & VEM & Autonomia & Competência & Relação \\
\hline Item1 (C) & $2-5$ & $3.89 \pm 0.64$ & 0.47 & - & - & - & - & - \\
Item2 (R) & $3-5$ & $4.27 \pm 0.56$ & 0.67 & - & - & - & - & - \\
Item3 (A) & $1-5$ & $4.25 \pm 0.66$ & 0.65 & - & - & - & - & - \\
Item4 (C) & $2-5$ & $4.04 \pm 0.63$ & 0.65 & - & - & - & - & - \\
Item5 (R) & $2-5$ & $4.17 \pm 0.69$ & 0.81 & - & - & - & - & - \\
Item6 (A) & $2-5$ & $4.20 \pm 0.60$ & 0.73 & - & - & - & - & - \\
Item7 (C) & $2-5$ & $3.75 \pm 0.75$ & 0.64 & - & - & - & - & - \\
Item8 (R) & $2-5$ & $3.96 \pm 0.70$ & 0.76 & - & - & - & - & - \\
Item9 (A) & $1-5$ & $3.98 \pm 0.68$ & 0.54 & - & - & - & - & - \\
Item10 (C) & $1-5$ & $4.03 \pm 0.68$ & 0.66 & - & - & - & - & - \\
Item 11 (R) & $1-5$ & $3.74 \pm 0.79$ & 0.67 & - & - & - & - & - \\
Item 12 (A) & $1-5$ & $4.04 \pm 0.69$ & 0.48 & - & - & - & - & - \\
Autonomia & $2-5$ & $4.12 \pm .471$ & - & .70 & .37 & 1 & - & - \\
Competência & $2-5$ & $3.92 \pm .482$ & - & .70 & .35 & .43 & 1 & - \\
Relação & $2-5$ & $4.03 \pm .550$ & - & .82 & .53 & .28 & .25 & - \\
Índice Global & $2-5$ & $4.02 \pm .391$ & - & .80 & .57 & - & - & - \\
\hline
\end{tabular}

Nota. FL = Fator Latente; FC = Fiabilidade Compósita; VEM = Variância Extraída Média 
Tabela 2.

\section{Exploração da Versão Portuguesa da BPNES}

Índices de ajustamento dos modelos de medida de $1^{\mathrm{a}}$ ordem testados (incluindo versões existentes)

\begin{tabular}{|c|c|c|c|c|c|c|c|c|c|}
\hline Modelos & $\chi^{2}$ & df & $\chi^{2} / \mathbf{d f}$ & $B-S p$ & SRMR & TLI & CFI & RMSEA & RMSEA-90\% \\
\hline M1 & $122.30 *$ & 51 & 2.40 & - & .03 & .97 & .97 & .05 & $.04-.06$ \\
\hline M2 & 144.1 & 51 & 2.82 & - & - & .94 & .95 & .06 & $.05-.07$ \\
\hline M3-G & 164.2 & 51 & 3.22 & $<.001$ & .04 & .92 & .94 & .06 & $.05-.07$ \\
\hline M4 -M & 103.7 & 51 & 2.03 & .018 & .05 & .92 & .94 & .06 & $.04-.07$ \\
\hline M5 -F & 109.2 & 51 & 2.14 & .006 & .06 & .92 & .94 & .06 & $.05-.08$ \\
\hline M6-MS & 76.9 & 51 & 1.51 & .058 & .06 & .93 & .95 & .06 & $.03-.08$ \\
\hline M7-AG & 100.2 & 51 & 1.96 & .022 & .07 & .90 & .92 & .07 & $.05-.09$ \\
\hline M8-CF & 106.3 & 51 & 2.08 & .004 & .06 & .90 & .92 & .07 & $.05-.09$ \\
\hline
\end{tabular}

. Comparative Fit Index; RMSEA = Root Mean Squared Error of Approximation; RMSEA-90\% = Root Mean Squared Error of Approximation e o respetivo intervalo de confiança; * * p<.01; - = valores não reportados pelos autores; M1= modelo original (Vlachopoulos \& Michalidou, 2006); M2= versão Portuguesa modificada (Moutão et al., 2012); M3= amostra geral-presente estudo; M4= amostra género masculino presente estudo; M5= amostra género feminino presente estudo; M6= amostra musculação presente estudo; M7= amostra aulas de grupo presente estudo; M8= amostra cardiofitness presente estudo.

Tabela 3.

Índices de ajustamento dos modelos de medida de $2^{\mathrm{a}}$ ordem testados (incluindo versões existentes)

\begin{tabular}{|c|c|c|c|c|c|c|c|c|c|c|}
\hline & Modelos & $\chi^{2}$ & df & $\chi^{2} / d f$ & $B-S p$ & SRMR & TLI & CFI & RMSEA & RMSEA-90\% \\
\hline M1 & & 209.9 & 51 & 4.12 & - & - & .97 & .98 & .06 & $.05-.07$ \\
\hline $\mathrm{M} 2$ & & 201.72 & 51 & - & - & - & .94 & .95 & .06 & $.04-.07$ \\
\hline M4 & & 103.7 & 51 & 2.01 & .018 & .05 & .92 & .94 & .06 & $.04-.07$ \\
\hline M5 & & 109.2 & 51 & 2.14 & .006 & .05 & .92 & .94 & .07 & $.05-.08$ \\
\hline M6 & & 76.9 & 51 & 1.51 & .186 & .06 & .93 & .95 & .06 & $.03-.09$ \\
\hline M7 & & 100.2 & 51 & 1.96 & .020 & .06 & .90 & .91 & .07 & $.05-.09$ \\
\hline M8 & & 106.3 & 51 & 2.01 & .006 & .05 & .90 & .92 & .07 & $.05-.09$ \\
\hline
\end{tabular}

Nota. $\chi^{2}=$ qui-quadrado; $\mathrm{df}=$ graus de Liberdade; $\chi^{2} / \mathrm{df}=$ qui-quadrado normalizado; B-Sp= bootstrap bollen stine; SRMR $=$ Standardized Root Mean Square Residual; TLI = Tucker-Lewis Index; CFI $=$ Comparative Fit Index; RMSEA = Root Mean Squared Error of Approximation; RMSEA-90\% = Root Mean Squared Error of Approximation e o respetivo intervalo de confiança; * = p <.01; - = valores não masculino presente estudo; M5= amostra género feminino presente estudo; M6= amostra musculação presente estudo; M7= amostra aulas de grupo presente estudo; M8; amostra cardiofitness presente estudo 


\section{Cid, Rodrigues, Teixeira, Alves, Machado, Murillo-Rodríguez, Monteiro}

Tabela 4.

Invariância do modelo de medida ( $1^{\mathrm{a}}$ ordem) da BPNESp entre género e modalidades de ginásio

\begin{tabular}{|c|c|c|c|c|c|c|c|}
\hline Modelos & $\chi^{2}$ & df & $\Delta \chi^{2}$ & $\Delta \mathrm{df}$ & $\mathrm{p}$ & CFI & $\Delta \mathrm{CFI}$ \\
\hline \multicolumn{8}{|l|}{ Masculino - Feminino } \\
\hline Invariância Configural & 212.868 & 102 & - & - & - & .937 & - \\
\hline Invariância Métrica & 226.678 & 111 & 13.809 & 9 & .129 & .935 & .002 \\
\hline Invariância Escalar & 232.041 & 117 & 19.173 & 15 & .206 & .935 & .002 \\
\hline Invariância Residual & 254.897 & 129 & 42.028 & 27 & .033 & .929 & .008 \\
\hline \multicolumn{8}{|c|}{ Musculação - Aulas Grupo } \\
\hline Invariância Configural & 183.268 & 102 & - & - & - & .932 & - \\
\hline Invariância Métrica & 201.632 & 111 & 18.364 & 9 & .014 & .925 & .007 \\
\hline Invariância Escalar & 205.608 & 117 & 22.340 & 15 & .017 & .926 & .006 \\
\hline Invariância Residual & 234.379 & 129 & 51.107 & 27 & .003 & .912 & .020 \\
\hline \multicolumn{8}{|c|}{ Musculação - CardioFitness } \\
\hline Invariância Configural & 183.268 & 102 & - & - & - & .932 & - \\
\hline Invariância Métrica & 201.632 & 111 & 18.634 & 9 & .031 & .925 & .007 \\
\hline Invariância Escalar & 203.608 & 117 & 22.340 & 15 & .099 & .926 & .006 \\
\hline Invariância Residual & 234.375 & 129 & 51.107 & 27 & .033 & .912 & .020 \\
\hline \multicolumn{8}{|c|}{ Aulas Grupo - CardioFitness } \\
\hline Invariância Configural & 206.571 & 102 & - & - & - & .920 & - \\
\hline Invariância Métrica & 214.632 & 111 & 8.060 & 9 & .528 & .919 & .001 \\
\hline Invariância Escalar & 219.218 & 117 & 12.647 & 15 & .630 & .919 & .001 \\
\hline Invariância Residual & 263.065 & 129 & 56.494 & 27 & .001 & .896 & .024 \\
\hline
\end{tabular}

Nota. $\chi^{2}=$ teste do qui-quadrado; $\mathrm{df}=$ graus de liberdade; $\Delta \chi^{2}=$ diferenças no teste do qui-quadrado; $\mathrm{p}=$ nível de significância; $\mathrm{CFI}=$ Comparative Fit Index; $\Delta \mathrm{CFI}=$ diferenças no CFI

\section{Tabela 5.}

Invariância do modelo de medida (2 $2^{\mathrm{a}}$ ordem) da BPNES entre género e modalidades de ginásio

\begin{tabular}{|c|c|c|c|c|c|c|c|}
\hline Modelos & $\chi^{2}$ & $\mathrm{df}$ & $\Delta \chi^{2}$ & $\Delta \mathrm{df}$ & $\mathrm{p}$ & CFI & $\Delta \mathrm{CFI}$ \\
\hline \multicolumn{8}{|l|}{ Masculino - Feminino } \\
\hline Invariância Configural & 212.868 & 102 & - & - & - & .937 & - \\
\hline Invariância Métrica & 226.678 & 111 & 13.809 & 9 & .129 & .935 & .002 \\
\hline Invariância Regressões & 229.635 & 114 & 16.767 & 12 & .159 & .935 & .002 \\
\hline Invariância Residual & 254.897 & 129 & 42.028 & 27 & .033 & .929 & .008 \\
\hline \multicolumn{8}{|c|}{ Musculação - Aulas Grupo } \\
\hline Invariância Configural & 177.164 & 102 & - & - & - & .929 & - \\
\hline Invariância Métrica & 189.754 & 111 & 12.590 & 9 & .182 & .926 & .003 \\
\hline Invariância Regressões & 191.544 & 114 & 14.380 & 12 & .277 & .925 & .004 \\
\hline Invariância Residual & 226.577 & 129 & 49.413 & 27 & .005 & .919 & .010 \\
\hline \multicolumn{8}{|c|}{ Musculação - CardioFitness } \\
\hline Invariância Configural & 183.268 & 102 & - & - & - & .932 & - \\
\hline Invariância Métrica & 201.632 & 111 & 18.645 & 9 & & .925 & .007 \\
\hline Invariância Regressões & 204.913 & 114 & 21.645 & 12 & & .924 & .008 \\
\hline
\end{tabular}

Cuadernos de Psicología del Deporte, 20, 2 (mayo) 


\section{Exploração da Versão Portuguesa da BPNES}

\begin{tabular}{|c|c|c|c|c|c|c|c|}
\hline Invariância Residual & 234.375 & 129 & 51.107 & 27 & & .912 & .020 \\
\hline \multicolumn{8}{|c|}{ Aulas Grupo - CardioFitness } \\
\hline Invariância Configural & 206.571 & 102 & - & - & - & .920 & - \\
\hline Invariância Métrica & 214.632 & 111 & 8.060 & 9 & .528 & .919 & .001 \\
\hline Invariância Regressões & 219.186 & 114 & 12.614 & 12 & .398 & .918 & .002 \\
\hline Invariância Residual & 263.065 & 129 & 56.494 & 27 & .001 & .912 & .008 \\
\hline
\end{tabular}

Nota. $\chi^{2}=$ teste do qui-quadrado; $\mathrm{df}=$ graus de liberdade; $\Delta \chi^{2}=$ diferenças no teste do qui-quadrado; $\mathrm{p}=$ nível de significância; $\mathrm{CFI}=$ Comparative Fit Index; $\triangle \mathrm{CFI}=$ diferenças no CFI

\section{Validade de constructo}

De uma forma descritiva, os resultados revelam que os praticantes têm tendência a valorizar os itens do questionário, tal como é demonstrado pelas médias moderadas e altas em todos os itens. Desta forma, evidenciam a importância teórica subjacente às três necessidades psicológicas básicas preconizada pela SDT (Ryan \& Deci, 2017). De forma empírica, os resultados estão em linha com outros estudos que utilizaram a BPNES, nomeadamente: a versão original (Vlachopoulos \& Michailidou, 2006); a versão portuguesa (Moutão et al., 2012) e; a versão espanhola (Moreno-Murcia, Galindo, Pérez, Marcos \& Borges, 2012). Verificou-se ainda, valores de correlação positivos e significativos entre os três fatores (autonomia, competência e relação), o que corrobora com as evidências verificadas em outros estudos (e.g., Moutão et al., 2012; Vlachopoulos, 2007; Vlachopoulos \& Michailidou, 2006). Estudos em diferentes contextos como na educação física (Cid et al., 2016) e no desporto (Monteiro et al., 2016) apresentaram valores semelhantes aos presentes neste estudo

No que concerne à análise das propriedades psicométricas do BPNESp, os resultados demonstraram um ajustamento razoável aos dados, evidenciando que a sua estrutura está de acordo com a versão portuguesa (Moutão et al., 2012), versão original (Vlachapoulos \& Michailidou, 2006), e outros estudos realizados na população grega (Vlachopoulos, 2007, 2008; Vlachopoulos \& Neikou, 2007), bem como com as diferentes versões da BPNES para o contexto do exercício: Inglaterra (Vlachopoulos et al., 2010), Espanha (Murcia et al., 2012) e Turquia (Vlachopoulos et al. 2013).
No seguimento da análise do modelo de medida, o modelo de $2^{\mathrm{a}}$ ordem testado também apresentou propriedades psicométricas idênticas à versão original (Vlachopoulos \& Michailidou, 2006) e à versão portuguesa (Moutão et al., 2012). Do ponto de vista empírico, estes resultados suportam a utilização de um fator global de $2^{\mathrm{a}}$ ordem para medir a satisfação das três necessidades psicológicas. De facto, esta tem sido uma estratégia utilizada por diversos investigadores nos mais variados contextos: trabalho (Deci et al., 2001); educação física (Ntoumanis et al. 2005); exercício (Wilson et al., 2009; Teixeira et al., 2018); Educação (Gagné, 2003) e Desporto (Monteiro et al., 2018).

Em suma, a utilização de um índice global parece ser uma estratégia recorrente, de forma a reduzir o número de parâmetros a estimar, mantendo, no entanto, a integridade teórica da SDT (Deci, et al., 2001; Ntoumanis, 2005; Vlachopoulos, 2007) fornecem um suporte empírico muito consistente quanto à utilização da BPNES na avaliação das necessidades psicológicas básicas, dando robustez empírica ao quadro conceptual preconizado por Deci e Ryan (1985).

Recordamos que de acordo com este modelo teórico, as necessidades psicológicas básicas são nutrientes ou condições essenciais para o crescimento e bem-estar psicológico, para que as pessoas possam desenvolver todo o seu potencial, assim como, sob o ponto de vista biológico, "as plantas necessitam de sol e água para crescerem" e "as pessoas necessitam de alimentos, água e abrigo para prosperar" (Ryan, 1995, p. 410). Por isso, os autores da SDT afirmam de forma convicta, que as três necessidades psicológicas básicas no seu conjunto (autonomia, competência e relação) são essenciais e negligenciar uma delas tem efeitos funcionais negativos (i.e. mal-estar psicológico) (Deci \& Ryan, 2000). E é por essa razão que os autores 


\section{Cid, Rodrigues, Teixeira, Alves, Machado, Murillo-Rodríguez, Monteiro}

afirmam de forma categórica: "esta questão tem sido bem sustentada, e no nosso ponto de vista está especialmente bem testada" (Ryan \& Deci, 2007, p. 14). De facto, se atendermos à investigação realizada no campo do desporto e do exercício, facilmente comprovamos neste caso concreto que a satisfação das necessidades psicológicas básicas promove o bemestar psicológico (e.g. Edmunds, et al., 2008; Gagné, et al., 2003; Reinboth \& Duda, 2006; Vlachopoulos \& Karavani, 2009; Wilson, et al., 2009).

No que à fiabilidade diz respeito, tanto os fatores de $1^{\text {a }}$ ordem como o fator global de $2^{\mathrm{a}}$ ordem revelaram valores acima de 0.70 , sendo assim considerados como aceitáveis de acordo com vários autores (Hair et al., 2014; Fornell \& Larcker, 1981).

No que se refere à validade convergente, os fatores autonomia e competência demonstraram problemas de validade convergente (VEM <.50). No entanto, o fator relação (VEM =.53) e o fator global de $2^{\mathrm{a}}$ ordem $(\mathrm{VEM}=.80)$ demonstraram valores aceitáveis de validade convergente. Além disso, todos os pesos fatoriais foram significativos nos respetivos fatores, $o$ que de acordo com Hair et al. (2014) é um indicador de validade convergente. Acresce ainda o facto de que não foram encontrados cross-loadings, sendo por isso igualmente um indicador de validade convergente (Byrne, 2010).

Relativamente à validade discriminante, o instrumento demonstrou um problema envolvendo a autonomia e competência, visto que valor de VEM ter sido inferior ao quadrado da correlação de ambos os fatores (Hair et al., 2014). Resultados semelhantes foram encontrados na versão espanhola (Murcia et al., 2012) e na versão portuguesa (Moutão et al., 2012), o que poderá ser justificado pela elevada correlação entre eles, tal como verificado em diversos estudos (e.g. Vlachopoulos, 2007, 2008; Vlachopoulos \& Michailidou, 2006). Em termos teóricos, Deci e Ryan (2000) afirmam que que quanto maior a perceção de autonomia do sujeito, maior a perceção de competência, e vice-versa.

\section{Invariância Métrica}

Relativamente à invariância em função do género e das diferentes atividades praticadas no ginásio (i.e., neste caso concreto entre praticantes de aulas de grupo, cardiofitness e musculação), tanto do modelo de $1^{\mathrm{a}}$ ordem como de $2^{\mathrm{a}}$ ordem, os resultados revelaram que os modelos foram invariantes. Desta forma, podemos afirmar o seguinte: que o mesmo conjunto de itens que explica o mesmo conjunto de fatores se mantem, independentemente da atividade praticada, verificando-se por isso invariância configural; todos os pesos fatoriais são invariantes em ambas as atividades, ou seja, os itens mostram a mesma importância para os fatores, independentemente da atividade praticada, verificando-se assim invariância métrica; os intercetos dos itens são equivalentes em ambas as atividades, isto é, invariância escalar. No caso do modelo de $2^{\mathrm{a}}$ ordem verificou-se invariância das regressões, ou seja, os pesos das regressões do fator de $2^{\mathrm{a}}$ ordem para os fatores de $1^{\mathrm{a}}$ ordem foram equivalentes entre género $\mathrm{e}$ diferentes atividades praticadas, de acordo com os pressupostos deste tipo de análise (Byrne, 2010).

Olhando para a invariância residual, este pressuposto não foi respeitado. Contudo, de acordo com alguns autores (e.g. Byrne, 2010; Cheung \& Rensvold, 2002), parece não haver consenso na literatura sobre a necessidade de avaliar a invariância residual, pelo que a avaliação deste pressuposto é considerada opcional pelo investigador, derivado ao facto de ser muito restritiva e difícil de alcançar na área das ciências sociais. No entanto, Byrne (2010) afirma que falta de invariância residual não implica a falta de invariância do modelo de medida, desde que os outros pressupostos sejam respeitados, tal como observando no presente estudo.

Relativamente a análise de invariância do BPNES, resultados semelhantes foram encontrados no contexto do exercício (Vlachopulos et al., 2013), na educação física (Cid et al., 2016), no desporto (Monteiro et al., 2016) e entre géneros em vários estudos (e.g., Vlachopoulos, 2008). Contudo, no que respeita ao modelo de $2^{\mathrm{a}}$ ordem, a literatura parece não apresentar evidências, uma vez que até à data nenhum estudo analisou a invariância do modelo de $2^{\mathrm{a}}$ ordem. No entanto, estes resultados vão de encontro com o quadro teórico da SDT, onde os seus autores (Deci \& Ryan, 2000) afirmam que as necessidades psicológicas básicas são inatas e universais ao ser humano, independentemente do género, etnia ou repertório cultural. 


\section{Exploração da Versão Portuguesa da BPNES}

\section{CONCLUSÕES}

Tomando em consideração os objetivos deste estudo, podemos concluir que o modelo de três fatores com 12 itens da versão Portuguesa da Basic Psychological Needs in Exercise Scale (BPNESp: Moutão et al., 2012) continua a ser robusto, uma vez que, para além de evidenciar índices bastante razoáveis de ajustamento numa nova amostra de praticantes de exercício físico, continua a ser consistente com a estrutura do modelo original (Vlachopoulos \& Michailidou, 2006).

Partindo da premissa conceptual de que o bem-estar psicológico só pode ser alcançado em plenitude com a satisfação da autonomia, competência e relação no seu conjunto (Deci \& Ryan, 2000; Ryan \& Deci, 2007), comprovou-se empiricamente, à semelhança de outros estudos no contexto da atividade física (e.g. Ntoumanis, 2005; Vlachopoulos, 2007), a hipótese colocada sobre a existência de um fator de $2^{\mathrm{a}}$ ordem, como indicador global da satisfação das necessidades psicológicas básicas. De facto, o modelo hierárquico $\left(1\right.$ fator de $2^{\mathrm{a}}$ ordem $\rightarrow 3$ fatores de $1^{\mathrm{a}}$ ordem e 12 itens) da BPNESp apresenta índices de ajustamento bastante aceitáveis, o que sustenta essa hipótese. A análise da invariância do modelo de medida revelou que o mesmo pode ser utilizado com elevado grau de validade e finalidade entre géneros e diferentes atividades praticadas no ginásio, tanto o modelo de $1^{\mathrm{a}}$ como o de $2^{\mathrm{a}}$ ordem.

Em suma, as evidências encontradas no presente estudo, confirmam a validade e dão suporte à utilização sem reservas da versão Portuguesa da BPNESp (Moutão et al., 2012), para a avaliação das necessidades psicológicas básicas (individualmente ou através de um índice global) em investigações futuras no domínio do exercício. A avaliação de um modelo de medida é sempre um processo com inúmeras dificuldades e que necessita de uma enorme quantidade de tempo, pelo que aconselhamos que sejam realizados futuros estudos com esta escala em outras atividades de ginásio e que sejam realizadas análises da invariância deste modelo de medida em função de outras variáveis ou culturas, de forma a torná-lo ainda mais robusto do ponto de vista psicométrico.

\section{APLICAÇÕES PRÁTICAS}

O grande contributo do presente estudo, para além de demonstrar a robustez psicométrica do questionário, tanto de um modelo de $1^{\text {a }}$ ordem como de um modelo de $2^{\mathrm{a}}$ ordem, consiste na sua aplicabilidade em praticantes de exercício físico em ginásios e health clubs, bem como de diferentes atividades de ginásio, o que pode ajudar os técnicos de exercício físico e diretores técnicos a avaliar as necessidades psicológicas básicas. A sua avaliação regular pode ajudar a prevenir o abandono, uma vez que quando as necessidades psicológicas básicas estão satisfeitas, existe uma maior motivação autónoma, resultando em consequências comportamentais, emocionais e cognitivas mais positivas (e.g., persistência, divertimento, prazer).

\section{REFERÊNCIAS}

Álvarez, M., Castillo, I., Duda, J., \& Balaguer, I. (2009). Clima motivacional, metas de logro e motivacion autodeterminada en futbolistas cadetes. Revista de Psicología General y Aplicada, 62(1-2), 35-44.

Byrne, B. (2010). Structural equation modeling with AMOS. Basic concepts, applications, and programming (2nd ed.) Madison, Avenue, New York: Taylor \& Francis Group, LLC.

Cheung, G., \& Rensvold, R. (2002). Evaluating goodness-of-fit indexes for testing measurement invariance. Structural Equation Modelling: A Multidisciplinary Journal, 9(2), 233-255. doi:10.1207/S15328007SEM0902_5

Cid, L., Stobaus, C., Monteiro, D., Moutão, J., Davoglio, T. \& Lettnin, C. (2016). Crosscultural validation of the basic psychological needs in physical education between Portugal and Brazil Samples. The Spanish Journal of Psychology, 19(5). doi: $10.1017 /$ sjp.2016.6

Deci, E., \& Ryan, R. (1985). Intrinsic Motivation and Self-Determination in Human Behavior. New York: Plenum Press. doi: 10.1016/B978-0-12-809324-5.05613-3

Deci, E., \& Ryan, R. (2000). The "What" and "Why" of Goal Pursuits: Human Needs and the Self-Determination of Behavior. 


\section{Cid, Rodrigues, Teixeira, Alves, Machado, Murillo-Rodríguez, Monteiro}

Psychological Inquiry, 11(4), 227-268. doi: 10.1207/S15327965PLI1104_01

Deci, E., Ryan, R., Gagné, M., Leone, D., Usunov, J., \& Kornazheva, B. (2001). Need Satisfaction, Motivation, and WellBeing in the Work Organizations of a Former Eastern Bloc Country: A CrossCultural Study of Self-Determination. Personality and Social Psychology Bulletin, 27(8), 930-942. doi: $10.1177 / 0146167201278002$

Edmunds, J., Ntoumanis, N., \& Duda, J. (2006). A Test of Self-Determination Theory in the Exercise Domain. Journal of Applied Social Psychology 36 (9): 2240-2265; 36(9), 2240-2265. doi: 10.1111/j.00219029.2006.00102.x

Edmunds, J., Ntoumanis, N., \& Duda, J. (2008). Testing a sel-determination theory-based teaching style intervention in the exercise domain. European Journal of Social Psychology, 38, 375-388. doi: 10.1002/ejsp.463

Fornell, C. \& Larcker, D. F. (1981). Evaluating structural equation models with unobservable variables and measurement error. Journal of Marketing Research, 18(1), 39-50. doi:10.2307/3151312

Gagné, M. (2003). The Role of Autonomy Support and Autonomy Orientation in Prosocial Behavior Engagment. Motivation and Emotion, 27(3), 199-223. doi: 10.1023/A:1025007614869

Gagné, M., Ryan, R., \& Bargmann, K. (2003). Autonomy support and need satisfaction in the motivation and well-being of gymnasts. Journal of Applied Sport Psychology, 15, 372-390. doi: 10.1080/714044203

Hagger, M., \& Chatzisarantis, N. (2008). Selfdetermination Theory and the Psychology of Exercise. International Review of Sport and Exercise Psychology, 1(1), 79-103. doi: 10.1080/17509840701827437

Hair, J., Black, W., Babin, B., \& Anderson, R., (2014). Multivariate Data Analysis (7th ed.). New Jersey: Pearson Educational, Inc.

Kahn, J. (2006). Factor Analysis in Counseling Psychology. Research, Trainning, and Practice: Principles, Advances and Applications. The Counseling Psychologist, 34(5), 684-718. doi: $10.1177 / 0011000006286347$

Markland, D., \& Tobin, V. (2010). Need support and behavioural regulations for exercise among exercise referral scheme clients: The mediating role of psychological need satisfation. Psychology of Sport and Exercise, 11, 91-99. doi: 10.1016/j.psychsport.2009.07.001

Marsh, H., Hau, K., \& Wen, Z. (2004). In search of golden rules: Comment on hypothesistesting approaches to setting cutoff values for fit indexes and dangers in overgeneralizing Hu and Bentler's (1999) findings. Structural Equation Modeling, 11(3), 320-341. doi: 10.1207/s15328007sem1103_2

Monteiro, D., Teixeira, D.S., Vitorino, A., Moutão, J., Rodrigues, F., Machado, S., \& Cid, L. (2019). Behavioral Regulation Sport Questionnaire: Gender and Sport Invariance in Portuguese Athletes. Perceptual and Motor Skills, 126(2), 323341. doi: 10.1177/0031512519825700.

Monteiro, D., Teixeira, D.S., Travassos, B., Duarte-Mendes, P., Moutão, J., Machado, S., \& Cid, L. (2018a). Perceived Effort in Football Athletes: The Role of Achievement Goal

Theory and Self-Determination Theory. Frontiers in Psychology 9: 1575. doi: 10.3389/fpsyg.2018.01575

Monteiro, D., Pelletier, L.G., Moutão, J., \& Cid, L. (2018b). Examining the motivational determinants of enjoyment and the intention to continue of persistent competitive swimmers. International Journal of Sport Psychology, 6(1), 49. Doi: 10.7352/IJSP.2018.49 


\section{Exploração da Versão Portuguesa da BPNES}

Monteiro, D., Marinho, D., Moutão, J., Couto, N., Antunes, R., \& Cid, L. (2016). Adaptation and validation of the Basic Psychological Needs Exercise Scale to the sport domain and invariance across soccer and swimming. Motricidade, 12(4), 51-61. doi.org/10.6063/motricidade.9372

Moreno Luque, M., Reigal Garrido, R. E., Morillo Baro, J. P., Morales Sánchez, V., \& Hernández Mendo, A. (2019). Estilo de interacción del entrenador, clima motivacional percibido y satisfacción de las necesidades psicológicas básicas en futbolistas jóvenes. Cuadernos De Psicología Del Deporte, 19(3), 79-89. doi: 10.6018/cpd.372381

Moreno-Murcia, J., Galindo, C., Pérez, V., Marcos, P. \& Borges, F. (2012). Confirmation of the Basic Psychological Needs in Exercise Scale (BPNES) with a sample who do healthy exercise. Journal of Sports Science and Medicine, 11(11), 141-146.

Moutão, J., Cid, L., Leitão, C., \& Alves, J. (2008). Tradução e Validação Preliminar da versão Portuguesa do Basic Psychological Needs in Exercise Scale (BPNESp). In J. Díaz, I. Díaz \& J. Dosil (Eds.), II Congresso da Sociedade Iberoamericana de Psicologia do Desporto. Consolidando la Psicología del Deporte Iberoamericana (pp. 314). Torrelavega, Espanã: SIPD.

Moutão, J., Cid, L., Leitão, C., Alves, J., \& Vlachopoulos, S. (2009). The Measurement of Basic Psychological Needs in Exercise. Confirmatory Factor Analysis of the Basic Psychological Needs in Exercise Scale (BPNESp) Portuguese Version. In A. Baria, H. Nabli, M. Madani, A. Essieedali, M. Aragon \& A. Ouartassi (Eds.), 12th World Congress of Sport Psychology (pp. 186). Marrakesh, Marroco: ISSP.

Moutão, J., Cid, L., Leitão, C., Alves, J., \& Vlachopoulos, S. (2012). Translation and Initial Validation of the Basic
Psychological Needs in Exercise Scale in a Portuguese Sample of Exercise Participants. The Spanish Journal of Psychology, 15 (1), 399-409. doi: 10.5209/rev_SJOP.2012.v15.n1.37346

Nevitt, J., \& Hancock, G. (2001). Performance of bootstrapping approaches to model test statistics and parameter standard error estimation in structural equation modeling. Structural Equation Modeling, $8(3)$, 353-377. doi:10.1207/S15328007SEM0803_2

Ntoumanis, N. (2001). A self-determination approach to the understanding of motivation in physical education. British Journal of Educational Psychology, 71, 225-242.

Ntoumanis, N. (2005). A Prospective Study of Participation in Optional School Psysical Education Using a Self-Determination Theory Framework. Journal of Educational Psychology, 97(3), 444-453. doi: 10.1037/0022-0663.97.3.444

Reinboth, M., \& Duda, J. (2006). Perceived motivacional climate, need satisfaction and indices of well-being in team sports: A longitudinal perspective. Psychology of Sport and Exercise, 7, 269-286. doi: 10.1016/j.psychsport.2005.06.002

Ryan, R. (1995). Psychological Needs and the Facilitation of Integrative Processes. Journal of Personality, 63(3), 397-427. doi: 10.1111/j.1467-6494.1995.tb00501.x Rodrigues, F., Hair, J., Neiva, H., Teixeira, D.S., Cid, L., \& Monteiro, D. (2019). The Basic Psychological Need Satisfaction and Frustration Scale in Exercise (BPNSFSE): validity, reliability, and invariance across gender. Perceptual and Motor Skills, 126 (5), 949-972. doi: 10.1177/0031512519863188.

Rodrigues, F., Bento, T., Cid, L., Neiva, H., Teixeira, D.S., Moutão, J., Marinho, D.A., \& Monteiro, D. (2018). Can interpersonal behavior influence the persistence and adherence to physical exercise practice in 


\section{Cid, Rodrigues, Teixeira, Alves, Machado, Murillo-Rodríguez, Monteiro}

adults? A systematic review. Frontiers in Psychology, 9: 2141.doi: 10.3389/fpsyg.2018.02141.

Rodrigues, F., Teixeira, D.S., Neiva, H.P., Cid, L., \& Monteiro, D. (2020). The bright and dark sides of motivation as predictors of enjoyment, intention, and exercise persistence. Scandinavian Journal of Medicine and Science in Sports. doi: $10.1111 / \mathrm{sms} .13617$.

Ryan, R., \& Deci, E. (2000). Self-Determination Theory and the Facilitation of Intrinsic Motivation, Social Development, and Well-Being. American Psychologist, 55(1), 68-78. doi:10.1037/0003066X.55.1.68

Ryan, R., \& Deci, E. (2002). Overview of SelfDetermination Theory: An Organismic Dialectial Perspective. In E. Deci \& R. Ryan (Eds.), Handbook of SelfDetermination Research (pp. 3-33). Rochester, New York: The University of Rochester Press.

Ryan, R., \& Deci, E. (2007). Active Human Nature: Self-Determination Theory and the Promotion and Maintenance of Sport, Exercise, and Health. In M. Hagger \& N. Chatzisarantis (Eds.), Intrinsic Motivation and Self-Determination in Exercise and Sport (pp. 1-19). Champaing, Illinois: Human Kinetics.

Ryan, R., Williams, G., Patrick, H., \& Deci, E. (2009). Self-determination theory and physical activity: The dynamics of motivation in development and wellness. Hellenic Journal of Psychology, 6(2), 107-124.

Sánchez J., \& Núñez, J. (2007). Análisis Preliminar da las Propiedades Psicométricas de la Versión Española de la Escala de Necesidades Psicológicas Básicas en el Ejercicio Físico. Revista Iberoamericana de Psicología del Ejercicio y el Deporte, 2(2), 83-92.

Standage, M., Gillison, F., \& Treasure, D. (2007). Self-Determination and Motivation in
Physical Education. In M. Hagger \& N. Chatzisarantis (Eds.), Intrinsic Motivation and Self-Determination in Exercise and Sport (pp. 71-85). Champaign, Illinois: Human Kinetics.

Teixeira, D., Silva, M., \& Palmeira, A. (2018). How does frustration make you feel? A motivational analysis in exercise context. Motivation and Emotion, 1-10. Doi: 10.1007/s11031-018-9690-6

Tristán, J., Torres, A., Farfano, M., Verdugo, F., Corrales, M., \& Tomás, I. (2019). Presentación de las tareas por el profesor, necesidades psicológicas y vitalidad subjetiva en alumnos de educación física. Cuadernos de Psicologia del Deporte, 19 (3), 190-204. doi: 10.6018/cpd.358561

Vallerand, R., Deci, E., \& Ryan, R. (1987). Intrinsic Motivation in Sport. In K. Pandolf (Ed.), Exercise and Sport Sciences Reviews (pp. 389-425). New York: MacMillan Publishing Company.

Vallerand, R., \& Losier, G. (1999). An Integrative Analysis of Intrinsic and Extrinsic Motivation in Sport. Journal of Applied Social Psychology, 11, 142-169. doi: 10.1080/10413209908402956

Vlachopoulos, S. (2007). Psychometric Evaluation of the Basic Psychological Needs in Exercise Scale in Community Exercise Programs: A Cross-Validation Approach. Hellenic Journal of Psychology, 4, 52-74.

Vlachopoulos, S. (2008). The Basic Psychological Needs in Exercise Scale: Measurement Invariance Over Gender. Structural Equation Modeling, 15, 114135. doi: 10.1080/10705510701758398

Vlachopoulos, S., \& Karavani, E. (2009). Psychological Needs and Subjective Vitality in Exercise: A Cross-Gender Situational Test of Needs Universality Hypothesis. Hellenic Journal of Psychology, 6, 207-222.

Vlachopoulos, S., \& Michailidou, S. (2006). Development and Initial Validation of a 


\section{Exploração da Versão Portuguesa da BPNES}

Measure of Autonomy, Competence, and Relatedness in Exercise: The Basic Psychological Needs in Exercise Scale. Measurement In Physical Education and Exercise Science, 10(3), 179-201.doi: 10.1207/s15327841mpee1003_4

Wilson, P., Mack, D., Blanchard, C., \& Gray, C. (2009). The Role of perceived Psychological Need Satisfation in Exercise-Related Affect. Hellenic Journal of Psychology, 6, 183-206.

\section{Agradecimentos}

Este estudo foi suportado pela Fundação para

Ciência e Tecnologia com o número de registo

UID/DTP/04045/2019 para os autores Luis Cid e Diogo Monteiro.

Este estudo também foi suportado pela Fundação para a Ciência e a Tecnologia, I.P., Projeto $\mathrm{N}^{\circ}$

UIDP/04748/2020 para o autor Filipe Rodrigues

Conflitos de interesse Nada a declarar. 